\section{C4 A CASE OF THE GREAT AND LITTLE IMITATOR}

doi:10.1136/sextrans-2012-050601b.4

${ }^{1} \mathrm{R}$ MacDonald, ${ }^{2} \mathrm{R}$ Fox. ${ }^{1}$ Sandyford Initiative; ${ }^{2}$ Brownlee Centre, Gartnavel General Hospital

We present a case of Acute Intermittent Porphyria (AIP) exacerbated by HAART and review the complications of managing these two conditions. Only one previous case of these two conditions has been published. $A B$ is a middle aged man who was diagnosed with HIV with baseline CD4 of 518. He was well until 2011 when he was admitted to our department with severe abdominal pain, hypotension and a penile ulcer. CT, MRCP, OGD and ultrasound were normal but syphilis serology was positive. He was treated with benzathine, his pain reduced and was discharged. He was then started on Atripla with a CD4 of 162. After 3 weeks he developed severe abdominal pain, hypertension and complained of 'bloody' urine but no blood on analysis. Biochemistry showed a sodium of 117. With these new symptoms urinary porphyrins were requested which were elevated at $4002 \mathrm{nmol} / 1$ representing an acute episode of AIP. It was felt this second admission was triggered by HAART which was stopped and he was treated with haem arginate and his symptoms improved. On further discussion with $A B$ he admitted to taking a 'legal high' prior to the first admission which may have triggered this episode. There have been no reported cases of AIP due to syphilis. AIP is a disorder of the haem pathway and is known as the Little Imitator in contrast to syphilis the Great Imitator as it is often misdiagnosed. While it is a rare condition its importance with HIV is that it is triggered by most drugs needed in managing HIV. Drugs which induce cytochrome p450, anti PCP, TB and fungal agents have all been implicated. NRTI's are thought to be safe while Saquinavir is the only PI recommended for use and there is no evidence of newer HIV agents. AB was started on Truvada and boosted Saquinavir and 4 months on his symptoms are controlled with no further AIP attacks and viral load is $42 \mathrm{c} / \mathrm{ml}$. Reports such as this are important in increasing the limited knowledge of safely co-treating HIV and AIP.

Correction notice This article has been corrected since it was published. The text has had minor edits made to protect patient anonymity.

\section{C5 NEUROLOGICAL COMPLICATIONS AS A SEROCONVERSION ILLNESS IN PRIMARY HIV INFECTION (PHI)}

doi:10.1136/sextrans-2012-050601b.5

${ }^{1} \mathrm{~L}$ Jain, ${ }^{* 1} \mathrm{~S}$ Lowe, ${ }^{2} \mathrm{G}$ Crowe. ${ }^{1}$ Barts and the Royal London NHS Trust, London, UK; ${ }^{2}$ The Princess Alexandra NHS Trust, Essex, UK

Background Neurological complications as the presenting feature of $\mathrm{PHI}$ are rare and reported in $<10 \%$ of cases. We describe two cases of sero-conversion illness with rapidly progressive neurological involvement.

Case 1 A 43-year-old man presented with diarrhoea, vomiting and rash. On examination he had widespread lymphadenopathy, mouth ulcers and tender hepatomegaly. HIV test was positive (avidity index 50\%). 2 weeks later he was readmitted with headache and vomiting. On examination he had left sided weakness and left facial droop. CT head was normal. Cerebro-spinal fluid (CSF) showed an elevated protein $2.99 \mathrm{~g} / \mathrm{l}$, WC 48 , with and numerous polymorphs visualised. Antibiotics for meningitis were commenced. Serial MRI scans were normal. CD4 count was $640 \mathrm{cell} / \mathrm{mm}$ and HIV viral load was 197000 . Antiretrovirals (ARV) were commenced at day 14 and symptoms improved within the next 4 days. 2 months later all weakness and headaches have resolved.

Case 2 A 47-year-old British man presented with a collapse following 2 days of personality change and confusion and 10 days of fever, headaches, diarrhoea, myalgia, photophobia and rash. On admission he was confused, pyrexial with a Glasgow Coma Score of 13. He had a normal CT head, CSF protein was $1.5 \mathrm{~g} / 1, \mathrm{RC}<5$, $\mathrm{WC}<5$, with no organisms seen. Subsequent CSF viral PCR was negative. MRI brain was also normal. No evidence of sepsis was identified. An HIV test on admission was positive (avidity index $65 \%$ ). HIV test 3 months earlier had been negative. CD4 count was $594(16 \%)$ cells $/ \mathrm{mm}^{3}$ and viral load 462756 copies $/ \mathrm{ml}$. ARVs were commenced on day 7, symptoms improved markedly over the following week. Four weeks post discharge confusion had completely resolved but he reported ongoing mild coordination difficulties.

Discussion Neurological complications of seroconversion are rare but do occur. Early recognition is important through prompt HIV testing and the introduction of ARVs may reduce the incidence of neurological sequelae markedly.

\section{C6} NOT ALL IRIS'S ARE EQUAL: A CASE OF SUDDEN DEATH doi:10.1136/sextrans-2012-050601b.6

E Wainwright, ${ }^{*}$ F Chen, A Tang. Royal Berkshire Hospital, Reading, UK

Background Myocarditis is a well-recognised complication of HIV infection but is seldom observed in clinical practice in the UK. Additionally, myocarditis has been associated with sudden unexplained death in the general population. The mode of death in these cases is likely to involve arrhythmias. We present a very rare case of sudden cardiac death related to HIV-associated myocarditis.

Case report A 47-year-old man was diagnosed with HIV in 2002. He was lost from follow-up between 2006 and 2009, when he represented with a CD4 of 10 . He commenced HAART in November 2009 and had a good response (CD4 70; viral load <50). In March 2010 he collapsed whilst playing football and a clinical diagnosis of cardiac arrest was made; resuscitation was unsuccessful. Retrospective history from a relative indicated no prodromal illness. A coroner's post-mortem examination indicated cardiac pathology as the most likely cause of death; this was supported by abnormal macroscopic appearance of the cardiac muscle. Histological examination of the myocardium showed focal fibrosis and lymphocytic infiltration. The finding of lymphocytic myocarditis in a previously asymptomatic and well person following 4 months of HAART strongly implicates immune reconstitution inflammatory syndrome (IRIS) myocarditis as the cause of the patient's death.

Conclusions The wide spectrum of IRIS is increasingly familiar to HIV physicians but this case illustrates the need to be aware of potentially fatal forms of IRIS with increased risk in the severely immunosuppressed. Some of these forms unfortunately have no easily discernible manifestations. 


\section{Corrections}

MacDonald R, Fox R. A case of the great and little imitator Sex Transm Infect 2012;88(Suppl 1):

A10. This text of this article has had minor edits made to protect patient anonymity.

Sex Transm Infect 2012;88:474. doi:10.1136/sextrans-2012-050601b.4corr1 